\title{
Neuro-Fuzzy Prediction of Cooperation Interaction Profile of Flexible Road Train Based on Hybrid Automaton Modeling
}

\author{
Lejla Banjanovic-Mehmedovic ${ }^{1}$, Nermin Delic ${ }^{2}$, Ivana Butigan ${ }^{3}$, Suad Kasapovic ${ }^{1}$ and Ivan Bosankic ${ }^{4}$ \\ ${ }^{1}$ Faculty of Electrical Engineering, University of Tuzla, 75000 Tuzla, Bosnia and Herzegovina \\ ${ }^{2}$ Magicsoft, 75000 Tuzla, Bosnia and Herzegovina \\ ${ }^{3}$ Leftor, 75000 Tuzla, Bosnia and Herzegovina \\ ${ }^{4}$ IVL, 75000 Tuzla, Bosnia and Herzegovina
}

\begin{abstract}
Accurate prediction of traffic information is important in many applications in relation to Intelligent Transport systems (ITS), since it reduces the uncertainty of future traffic states and improves traffic mobility. There is a lot of research done in the field of traffic information predictions such as speed, flow and travel time. The most important research was done in the domain of cooperative intelligent transport system (C-ITS). The goal of this paper is to introduce the novel cooperation behaviour profile prediction through the example of flexible Road Trains useful road cooperation parameter, which contributes to the improvement of traffic mobility in Intelligent Transportation Systems. This paper presents an approach towards the control and cooperation behaviour modelling of vehicles in the flexible Road Train based on hybrid automaton and neuro-fuzzy (ANFIS) prediction of cooperation profile of the flexible Road Train. Hybrid automaton takes into account complex dynamics of each vehicle as well as discrete cooperation approach. The ANFIS is a particular class of the ANN family with attractive estimation and learning potentials. In order to provide statistical analysis, RMSE (root mean square error), coefficient of determination (R2) and Pearson coefficient (r), were utilized. The study results suggest that ANFIS would be an efficient soft computing methodology, which could offer precise predictions of cooperative interactions between vehicles in Road Train, which is useful for prediction mobility in Intelligent Transport systems.
\end{abstract}

\section{Introduction}

Cyber-physical systems (CPSs) feature a tight combination and coordination between the system's computational and physical elements and integration of computer and information-centric physical and engineered systems [1]. Intelligent Transport systems (ITS) fall in the framework of cyber-physical systems due to the interaction between physical systems (vehicles) and a distributed information gathering and dissemination infrastructure. The transportation sector is responsible for a large part of the world's energy consumption and greenhouse gas emissions [2]. This is especially important if it is expected that the freight transport will increase considerably in the coming years. This provides a strong motivation for developing efficient road transportation sector, especially a cooperative approach, since road transportation is currently mainly done by individual vehicles.

The ITS rely on understanding, predicting and affecting the interactions between vehicles. The cooperative intelligent transport system (C-ITS) includes two or more actors working towards a common or mutually beneficial goal, purpose, or benefit; enabled by interaction and information exchange between the actors. Within C-ITS, information is shared between many actors such as vehicles, infrastructures, cloud services, etc. However, only sharing information is not enough to be considered a C-ITS, cooperation, and interaction between the actors in the system is also required [3].

The vehicle platoon is an example of cooperative intelligent transport system, where the desired behaviour of a vehicle is generally defined by a desired distance to the previous vehicle in the platoon [4]. Platoon is a control system, in which one vehicle - a leader, gives instructions to other vehicles - followers in form of an acceleration, deceleration, or joining/leaving a Platoon. A vehicle platoon can be defined as a set of vehicles that move together while keeping a predefined geometrical configuration without any material coupling. The most widely studied platoon configuration is the column, also known as train configuration, Road Train (RT) [5]. Road Train has many advantages, from increasing traffic density and safety, to simultaneously decreasing fuel consumption and driver tiredness [6].

A very basic Road Train is equipped with an Advanced Cruise Control (ACC) system, and the Cooperative Adaptive Cruise Control (CACC) systems are developed as an extended cruise control. These systems are able to react to vehicles which are located in front of them, and are capable to communicate with the vehicles located in front and in the back [7]. However, both ACC 
and CACC systems are never designed to function as a part of a Road Train or to increase road capacity. The longitudinal algorithms used in CACC systems are not well suited for Road Train: shock wave effects and string instability are likely to occur. In order to tackle this problem, Road Train systems were designed so that they would be capable to communicate not only with the vehicle located in front of them, but so that they could also gather information about the behaviour and aspects of the platoon as a whole. The final flexible Road Train can overcome these problems, whereas smart vehicles with artificial intelligence could merge and leave or enter the platoon whenever the driver wants.

Road Train models can be found in many systems [8], ranging from systems which do not include communication between the vehicles to systems which use full communication between the vehicles. There exists physics-inspired model of the Road Train: [8] it represents the interactions as virtual spring-damper systems, [9] it considers the Road Train as a multi-agent system, which mimics animal interaction behaviour, while [10] modelling the forces between the vehicles as Newton forces.

There is a lot of research in the field of traffic information predictions such as speed, flow and travel time [11]-[13]. Vehicular velocity data have proven to be very useful in many contexts, including the understanding of road usage patterns in urban areas [14].

Various techniques, e.g. neural networks, wavelet, neuro-fuzzy could be adopted to develop a parameter estimation model. The artificial neural network (ANN) can be used as an analytical method for various prediction purposes because it provides the benefit of independency on the knowledge of internal system parameters, compressed compact solution in terms of multi-variable problems and rapid computation [15]. The neural network prediction of the speed profile is presented in [11], [12]. The speed profile neural network based prediction for selected agents using vehicle-tovehicle (V2V) communication is presented in [13].

Amongst the many neural network systems, one of the most commonly used and powerful ones is the adaptive neuro-fuzzy system (ANFIS) [16]. ANFIS is a hybrid intelligent system that increases the capability of automatically learning and adapting.

The goal of this paper is to introduce the novel cooperation behaviour profile prediction through the example of the flexible Road Train, as useful road cooperation parameter which contributes to the improvement of traffic mobility in Intelligent Transportation Systems. Our methodology consists of flexible Road Train cooperation modelling based on hybrid automaton and ANFIS predictive model which has proven to have the quality for prediction of cooperative behaviour profile of the flexible Road Train.

This paper is organized as follows: Section 2 presents proposed hybrid automaton modelling framework of the flexible Road Train; Section 3 presents the cooperation interactions profile, using proposed behaviour pattern coding scheme. Section 4, presents the neuro-fuzzy prediction of the cooperation interactions profile. Finally, we give conclusions and directions for future work.

\section{Flexible road train modeling}

The Road Train is often modelled as a platoon of identical vehicles. We assume that each vehicle has the ability to communicate with other vehicles and is able to send and retrieve data content. The Road Train is formed so that each agent can be in one of the next modes: leader or follower in Road Train. The autonomous vehicles can use three manoeuvres: join, merge and leave the Road Train.

We model the Road Train as a hybrid system which exhibits a combination of discrete and continuous behaviour [17]. Hybrid automaton takes into account complex nonlinear dynamics of each vehicle and discrete cooperation approach. Model of a flexible Platoon consists of the following modules, presented on Fig. 1: Leader Vehicle Module, Cooperative Module, Control Module of Followers and Behaviour Scenario Module.

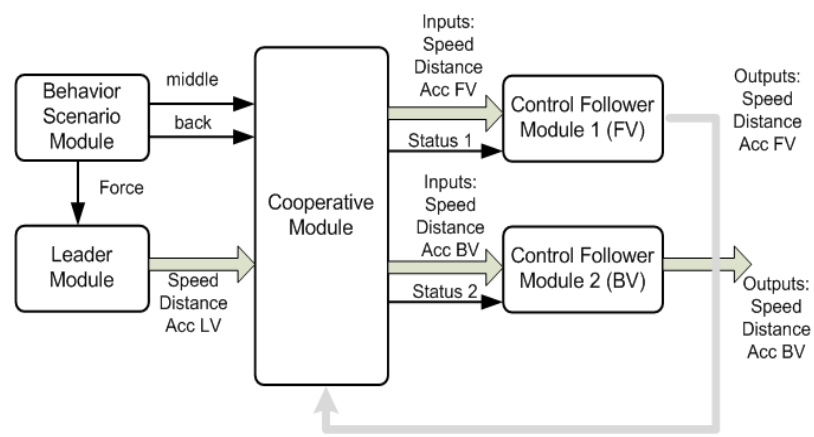

Figure 1. Block-diagram of flexible road train model.

Leader Module consists of model leader's vehicle dynamic. Cooperative Module is realized through the finite state machine (FSM) and together with Control Modules of Followers defines a hybrid approach in vehicle dynamics system. The Behaviour Scenario Module is realized using finite state machine, and it selects the parameters for different behaviours of the vehicles in the Road Train, as well as the speed changing. This module gives different case scenarios for the possibilities for joining, merging and leaving the follower vehicles from the Road Train in time.

\subsection{Vehicle modeling}

When it comes to vehicle modeling, we assume mathematical model of longitudinal motion of the vehicle [6], with two coordinated systems (vehicle-fixed coordinate system, $B(C ; x, z)$, and Earth-fixed coordinate system, $E\left(O ; x_{0}, z_{0}\right)$.

Application of Newton's second law on vehicle moving in the $x$ and $z$ directions, effects of aerodynamic force $D_{A}$ and the rolling resistance force $R_{x}$, give the next equation:

$$
m \dot{u}=F_{x}-m g \sin \theta-f_{r} m g \cos \theta-\frac{1}{2} C_{a i r}\left(u+u_{w}\right)^{2}
$$

where $\mathrm{m}$ is the vehicle mass, $g$ is the gravitational constant; $G=m g$ is the weight of the vehicle; $F_{x}$ is the tractive force, $u$ is the vehicle-forward velocity, $u_{w}$ is the wind velocity, $\theta$ is inclined upon angle with respect to horizontal plane (slope of the road) and constant $C_{\text {air }}$ 
(depends of the air density and the drag coefficient). The subscripts $r$ refer to the rear tire-reaction forces. Module leader is the block that represents the implementation of leader vehicle dynamics. The input variable is a force and the outputs are the speed, the acceleration, and the velocity.

\subsection{Control module of follower vehicles}

Under assumption that the dynamic behaviour of the object with respect to the nominal trajectory is linear, we can apply methods for synthesis developed for linear systems: proportional-integral-derivative (PID) controller design, Linear Quadratic Regulator (LQR), adaptive optimal control etc. In this paper, a (PID) control design approach is used in scope of Control Modules of Followers: first (FV) and second vehicle (BV). We generate reference acceleration $a_{0}$, velocity $v_{0}$ and position $x_{0}$ of the Road Train leader. These values go through Cooperation Module and then to the PID controller where they are processed according to [6]:

$$
u=\Delta F_{x}=K_{P}\left(x_{0}-x\right)+\frac{K_{I}}{s}\left(x_{0}-x\right)+K_{D}\left(v_{0}-v\right)
$$

where $x$ and $v$ are position and speed of each follower vehicles and $K_{P}, K_{I}$, and $K_{D}$ are proportional, integral and derivative gains of the PID controllers for follower vehicles.

The movement of vehicles is in the absolute coordinate system $G\left(O ; x_{o}, y_{o}\right)$, which is fixed to the road with origin in the starting point, $O$. Positions $x_{i}$, velocities $v_{i}$, and accelerations $a_{i}$ are measured with respect to $G$ $\left(0 ; x_{o}, y_{o}\right)$. Coordinate system $L\left(L ; x_{L}, y_{L}\right)$ is fixed to the vehicle-leader with origin in the centre of its mass. Using vehicle model (1), $\theta=0$ and $u_{w}=0$, we can find acceleration of the vehicle in this form:

$$
\begin{gathered}
\dot{u}=a=\frac{1}{m}\left(F_{x}-f_{r} m g-\frac{1}{2} C_{a i r} u^{2}\right) \\
F_{x}=\Delta F_{x}+F_{x 0}
\end{gathered}
$$

Control force $F_{x}$ is determined by a PID controller, i.e. with equation (2). Substituting (2) in (3), it is possible generate the acceleration for the $i$-th vehicle. The following equations represent linear state space model of the $i$-th vehicle in the platoon:

$$
\begin{gathered}
\dot{x}_{i}=v_{i} \\
\dot{v}_{i}=a_{i} \\
\dot{a}_{\iota}=\frac{1}{m}\left[\begin{array}{c}
\left.K_{I i}\left(x_{i-1}-x_{i}-h d_{i}\right)+K_{P i}\left(v_{i-1}-v_{i}\right)+\right] \\
K_{D i}\left(a_{i-1}-a_{i}\right)-C_{a i r} u^{0} a_{i}
\end{array}\right]
\end{gathered}
$$

where $h d_{i}$ is constant distance between $i$-1-th and $i$-th vehicle, variables $x_{i-1}, v_{i-1}, a_{i-1}$ of the previous ( $i-1-$ th vehicle) are input variables for the $i$-th vehicle.

\subsection{Cooperative module}

Cooperative Module gets the following as inputs: parameters of the joining, merging and/or leaving of individual follower vehicles from Behaviour Scenario Module, and speed and distance of the leader and first follower. Based on this data, this module makes the settings of status flag and relative distance, speed and acceleration of individual followers by specific joining, merging and/or leaving manoeuvres.

The finite state machine (FSM) of cooperation between the leader and two followers in the Road Train is presented in Fig. 2. It consists of 5 states: Only $L V$ on, $F V$ on, FV on BV on, FV off BV on and FV joining BV on.

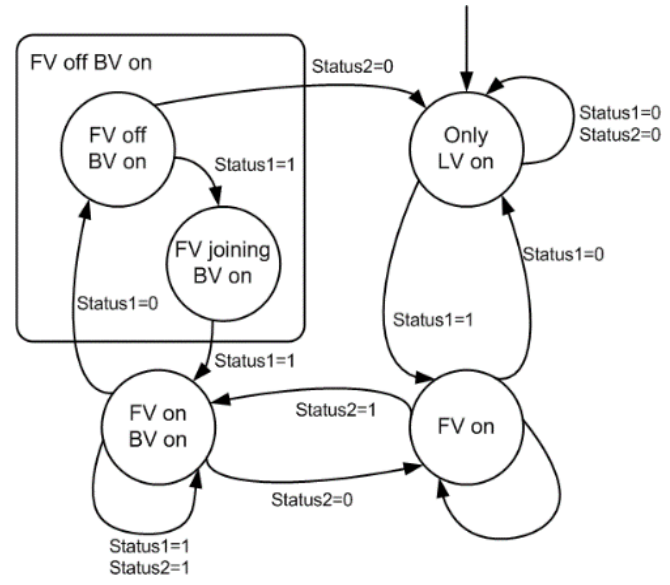

Figure 2. FSM of flexible road train.

The transitions between states are defined with value flags Status 1 and Status 2. The first state is the default state (Only leader on), where the only leader vehicle is in the Road Train. From this state, it is possible to make transition with new event to the state, where the first follower joins the Road Train ( $F V$ on). The Road Train (RT) remains in this state until it gets a signal for the inclusion of another follower or the exclusion of the first follower vehicle. In the first case, the transition leads to the state ( $F V$ on $B V$ on) in which both follower vehicles are in the Road Train. In the second case, the transition leads to the state (Only leader on), where both follower vehicles are excluded. From the state ( $F V$ on $B V$ on), the two transitions with new events are possible toward other states: toward the state ( $F V$ off $B V$ on $)$ which excludes the first follower, and toward the state $(F V$ on $)$, where the second follower is excluded. From the state ( $F V$ off $B V$ $o n)$, there are two possible transitions: excluding of another vehicle, transition to the state (Only LV on), or merging the FV vehicle to the Road Train, transition to the state (FV joining BVon) and ( $F V$ on BV on).

\section{Cooperation interaction profile}

We propose the coding of cooperation interactions between the vehicles in the Road Train toward cooperation profile. The coding scheme transforms more behaviour patterns in time into specific sum of activities in Road Train, presented in Table 1. In this way, we describe the complex system interactions only with one variable, which we use for tracking and predicting mobility in ITS. In case of more vehicle agents in the Road Train, we can use another coding scheme, for example binary coding. 
Table 1. Coding of cooperation interactions in road train.

\begin{tabular}{|c|c|c|}
\hline $\begin{array}{l}\text { Notation of } \\
\text { behaviour }\end{array}$ & $\begin{array}{c}\text { Sum of } \\
\text { behaviour } \\
\text { activities in } \\
\text { time }\end{array}$ & Explanation \\
\hline 1 & 1 & Only LV included \\
\hline 3 & $1+2$ & LV and FV included \\
\hline 4 & $1+3$ & LV and BV included \\
\hline 5 & $1+4$ & LV deceleration \\
\hline 6 & $1+2+3$ & All vehicles included \\
\hline 7 & $1+2+4$ & LV and FV deceleration \\
\hline 8 & $1+3+4$ & LV and BV deceleration \\
\hline 11 & $1+2+8$ & LV and FV acceleration \\
\hline 12 & $1+3+8$ & LV and BV acceleration \\
\hline 10 & $1+2+3+4$ & All vehicles; deceleration \\
\hline 14 & $1+2+3+8$ & All vehicles; acceleration \\
\hline
\end{tabular}

The Road Train (RT) control and cooperation model presented in this paper are demonstrated by means of simulation scenarios, which consider several situations that the vehicles in the RT may commonly encounter. All vehicles are the same with numerical values: $u^{0}=20 \frac{\mathrm{m}}{\mathrm{s}}$, $\theta^{0}=0, m=1000 \mathrm{~kg}, \rho=1,2 \frac{\mathrm{kg}}{\mathrm{m}^{3}}, A_{f}=1,2 \mathrm{~m}^{2}, C_{d}=$ $0,5, f_{r}=0,01, g=9,81 \frac{\mathrm{m}}{\mathrm{s}}, u_{w}=0, C_{\text {air }}=1,2$.

The desired distances among vehicles are $d_{x i 0}=100 \mathrm{~m}$. Parameters of PID controllers are: $K_{P i}=100, K_{I i}=$ $400, K_{D i}=200$.

The vehicles speed profiles and generated cooperation profile of joining/merging/leaving of followers vehicles in the RT are highlighted in Fig. 3 respectively. On the basis of the road cooperation behaviour profile, we can track the activities of all the vehicles in the RT in time.
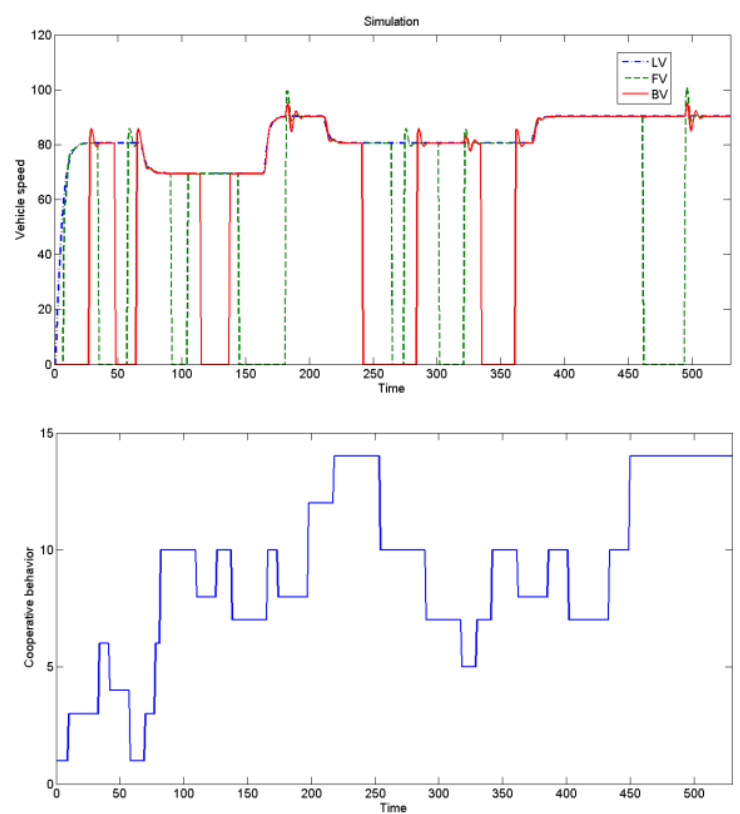

Figure 3. Vehicles speed and cooperation interactions profiles.

\section{Adaptive neuro-fuzzy prediction}

In this study, the ANFIS model was used to estimate the cooperative interactions profile in relation to the speeds of the leader and first and second follower vehicles. The main core of the ANFIS is the fuzzy inference system
(FIS). The FIS is funded on the basis of expertise described in the 'IF-THEN' rules. The fundamental organization of FIS includes: a rule base of a fuzzy rules selection, a database that establishes the membership functions (MFs) employed in the fuzzy rules and the reasoning mechanism to extract a rational output product. The method can be utilized to estimate the characteristics of various systems incorporating the uncertainty. The ANFIS combines the power of the FIS with a neural network back-propagation learning algorithm. The ANFIS structure consists of 5 layers. Fig. 4 shows the ANFIS structure with three inputs.

The first-order Sugeno model, including the fuzzy IFTHEN rules of the Takagi and Sugeno's class, and three inputs were all employed in our study:

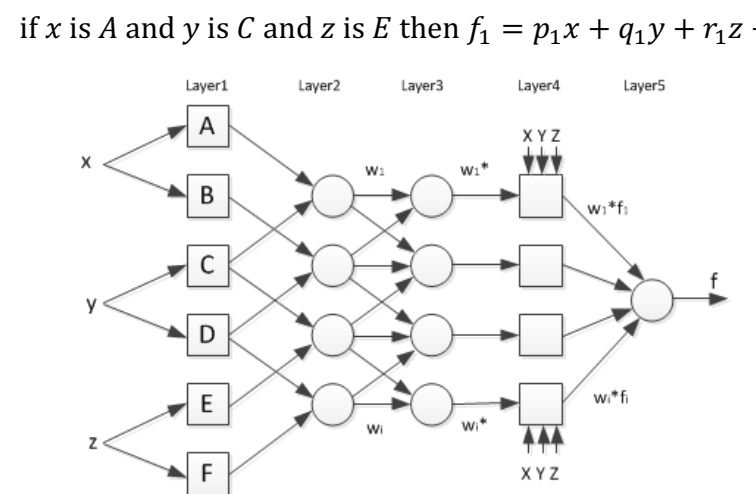

Figure 4. ANFIS structure.

The $1^{\text {st }}$ layer is comprised of input parameters membership functions (MFs). This layer provides the input quantities to the proceeding layer. Within this layer, each node is considered as adaptive with node function, $O=\mu(i)$, where $\mu(i)$ is the membership function. The parameters of this layer are designated as premise parameters. The $2^{\text {nd }}$ layer is also termed as the membership layer, and it monitors the MFs' weight. Each node in this layer is considered as non-adaptive. This layer acts as a multiplier for the receiving signals and it produces outcomes $w_{i}=\mu(i)_{i} \mu(i)_{i+1}$. Each node output represents the firing strength of a weight or rule.

The $3^{\text {rd }}$ layer is known as the rule layer. Here, all neurons act as the pre-condition matching the fuzzy rules. The nodes in the $3^{\text {rd }}$ layer are considered non-adaptive. Each of the nodes computes value of the rule's firing strength over the sum of all rules' firing strengths in the form of $w_{i}^{*}=\frac{w_{i}}{\sum_{i} w_{i}}$. The outcomes are refered to as the normalized firing strengths. The $4^{\text {th }}$ layer (known as the defuzzification layer) is responsible for providing the output values as a result of the inference of rules. Every $4^{\text {th }}$ layer node is an adaptive node having the node function: $O_{i}^{4}=w_{i}^{*} \cdot f=w_{i}^{*}\left(p_{i} x+q_{i} y+r_{i} z+t\right)$. In this layer, the $\left\{p_{i}, q_{i}, r_{i}, t\right\}$ is the variable set. The variable set is designated as the consequent parameters. The $5^{\text {th }}$ and final layer is known as the output layer. It adds up all the receiving inputs from the preceding layer. Thereafter, it converts the fuzzy classification outcomes into a binary (crisp). The single node of the $5^{\text {th }}$ layer is considered nonadaptive. This node calculates the total output as the whole sum of all receiving signals 


$$
O_{i}^{5}=\sum_{i} w_{i}^{*} \cdot f=\frac{\sum_{i} w_{i} f}{\sum_{i} w_{i}}
$$

In this prediction application, hybrid learning algorithms were employed to identify the variables within the ANFIS model. In the forward pass of the hybrid learning algorithm, the functional signals are passed forward until Layer 4. Moreover, consequent parameters are calculated using the least squares estimate. In the backward pass, the error rates propagate backwards and the premise parameters are updated by the gradient descent.

The input and output values are gathered according to the experimental scenarios in Road Train model. In order to evaluate the performance of ANFIS as a prediction model, it is necessary to have a rich dataset to experiment with. We used 3201 samples from behaviour scenario of the Road Train model. The leader, first and second follower's speeds from the Road Train model are used as input parameters and the generated cooperation behaviour profile is used as output parameter of ANFIS. In this study, $50 \%$ of the data was used to test the samples, while the other $50 \%$ was used for samples training.

As a data-driven model, the ANFIS ability for rational estimations is typically reliant on the choice of the ANFIS parameters. Therefore, the factors controlling the system (training epoch number, training error goal, step size rate, number and shapes of MFs) should be considered in the developing of a reliable network.

There are various ways to assess the performance of a prediction model. For the evaluation of the quality of predictors, we used several measures: RMSE (root mean square error), coefficient of determination $\left(\mathrm{R}^{2}\right)$ and Pearson coefficient ( $\mathrm{r}$ ). The best result of $\mathrm{s}$ prediction is using $\mathrm{R}^{2}=0.99$, $\mathrm{RMSE}=0.155$ and $\mathrm{MSE}=0.02$ for 7 Gauss membership functions for each input variables in 10 epochs. The comparison of predicted and cooperative behaviour from Road Train model is presented on Fig. 5.

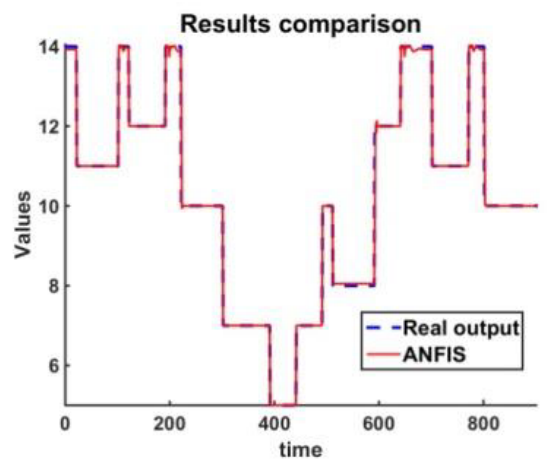

Figure 5. Prediction results of cooperation interactions profile.

\section{Conclusion and future work}

In this study, the Road Train hybrid automaton model was developed to simulate control and cooperation interactions between the vehicles. We introduced the coding scheme of behaviour patterns in the Road Train, for a specific cooperation interaction profile, in order to describe the complex system interactions only with one variable, which are useful for the prediction of traffic mobility in ITS. The results from ANFIS prediction model of cooperative interactions in Road Train showed the feasibility of this approach in terms of performance evaluation in the prediction method.

Future work will be devoted to exploring different prediction models, in order to test their performance in terms of influence on the vehicle speed profiles in the cooperation profile of the Road Train.

\section{Acknowledgment}

The authors express their sincere thanks for the funding support they received from Ministry of Civil Affairs of Bosnia and Herzegovina.

\section{References}

1. I. Stojmenovic, "Machine-to-Machine Communications With In-Network Data Aggregation, Processing, and Actuation for Large-Scale CyberPhysical Systems", IEEE Internet of Things Journal, 1(2), (2014)

2. B. Besselink, V. Turri, S.H. van de Hoef, K.-Y. Liang, A. Alam, J. Martensson, K.H. Johansson, "Cyber-physical Control of Road Freight Transport", (2015)

3. M. Aramrattana, T. Larsson, J. Jansson, C. Englund, "Dimensions of Cooperative Driving, ITS and Automation", IEEE Intelligent Vehicles Symposium (IV), Korea, (2015)

4. A. Ali, G. Garcia, P. Martinet, "The Flatbed Platoon Towing Model for Safe and Dense Platooning on Highways", Intelligent Transportation Systems Magazine, IEEE, 7(1) (2015)

5. M. El-Zaher, B. Dafflon, F. Gechter, J.-M. Contet, "Vehicle platoon control with multi-configuration ability", Procedia Computer Science, 9, 1503 (2012)

6. Z. Gacovski, S. Deskovski, "Different Control Algorithms for a Platoon of Autonomous Vehicles", International Journal of Robotics and Automation (IJRA), 3(3), 151 (2014)

7. G. Tillema: "Merging into a Demo 2000 Platoon", University of Twente, The Netherlands (2006)

8. D. Yanakiev, I. Kanellakopoulos, "A simplified framework for string stability analysis in AHS,"Proc. 13th IFAC World Congr., 177 (1996)

9. G. Franck, C. Vincent, C. Francois, "A reactive multi-agent system for localisation and tracking in mobile robotics,"Proc. 16th IEEE Int. Conf. Tools Artificial Intelligence, 431 (2004)

10. F. Contet, P. Gruer, A. Koukam, "Application of reactive multiagent system to linear vehicle platoon,"Proc. IEEE Conf. Tools Artificial Intelligence, Patras, Greece, 67 (2007)

11. E. Lee, J. Kim, W. Yoon, "Traffic speed prediction under weekday, time, and neighboring links' speed: Back propagation neural network approach". InLecture Notes in Computer Science. Berlin, Germany: Springer-Verlag, 626 (2007)

12. J. Park, Y.L. Murphey, R. McGee, J.G. Kristinsson, M.L. Kuang, A.M. Phillips, "Intelligent Trip Modeling for the Prediction of an Origin-Destination 
Traveling Speed Profile", IEEE Transactions on Intelligent Transportation Systems, 15 (2014)

13. I. Bosankic, L. Banjanovic-Mehmedovic, F. Mehmedovic: "Speed Profile Prediction in Intelligent Transport Systems Exemplified by Vehicle to Vehicle Interactions", Cybernetics and Information Technologies, Special Issue on Control in Transportation Systems, 15(5), (2015)

14. P. Wang, T. Hunter, A.M. Bayen, K. Schechtner, M.C. Gonzalez, "Understanding road usage patterns in urban areas", Nature Scientific Reports, 2 (2012)

15. X. Yang, M. Behroozi, O. A. Olatunbosun, "A Neural Network Approach to Predicting Car Tyre
Micro-Scale and Macro-Scale Behaviour". Journal of Intelligent Learning Systems and Applications, 6, 11 (2014)

16. V. Nikolic, S. Shamshirband, D. Petkovic, K. Mohammadi, Z. Cojbasic, T. A. Altameem, A. Gani, "Wind wake influence estimation on energy production of wind farm by adaptive neuro-fuzzy methodology", Elsevier Energy, 1 (2014)

17. N. Lynch, R. Segala, F. Vaandrager, "Hybrid I/O automata, Information and Computation" 185, 105 (2003). 